\title{
Welcome Address ESNR / ICS / SSNR September 13-16, 2006, Geneva - Switzerland
}

\author{
Dear members of ESNR \\ Dear attendees of ESNR / ICS / SSNR \\ Dear guests and friends
}

It is a privilege and honour for us to host in Geneva the 31st annual meeting of the European Society of Neuroradiology (ESNR'06). The meeting is held together with the 3rd International Intracranial Stent (ICS'06) meeting and the 18th Meeting of the Swiss Society for Neuroradiology (SSNR'06). The congress venue at the Hotel President Wilson is in the center of Geneva with a beautiful lakefront view. The choice of the venue will allow for associating hopefully memorable pictures of your visit to Geneva along with an exciting and balanced scientific programme that focuses on neurovascular diseases and CNS tissue characterization delivered in parallel sessions throughout the whole week for attendees more interested in diagnostic capabilities or in minimally invasive treatment guidance offered by medical imaging, enhanced by contrast materials and medical informatics or complemented by medical devices.

\section{Neurovascular Diseases}

The ESNR 06, 15th Advanced Course on September 13 will provide education on aneurysms and stroke. The use and role of modern neuroimaging modalities given with MR (diffusion, perfusion and angiography), CT (perfusion and angiography), and DSA (3DRA, treatment planning) techniques will be addressed and discussed. Concerning aneurysms, the contribution of minimally invasive treatment (MIT) modalities provided by interventional neuroradiological techniques are presented in view of ISAT (International Subarachnoid Aneurysm Trial), the most substantial contribution of ESNR members toward recognition of endovascular treatment for cerebral aneurysms at the international level. This part is in common with the ICS'06 meeting. The advanced course will in the afternoon session address and discuss neuroradiological contributions to stroke imaging and treatment. These sessions will mainly focus on non-invasive ways to investigate cerebral vascular diseases by using CT perfusion and MR diffusion and perfusion techniques in the acute assessment of ischemia.

The ICS 06, 3rd international annual meeting will run parallel to the Advanced Course and ESNR during September 1315. During these days, it will provide the platform for interventional neuroradiology issues centred on cerebral aneurysms and novel minimally invasive treatment possibilities using intracranial stents. Intracranial stents are the next generation medical devices that will allow for a more thorough minimally invasive endovascular repair of cerebral vessels by correcting abnormal local flow conditions. Pathological flow conditions exist in patients with an aneurysm and depend on multiple factors such as e.g. geometrical configuration of the aneurysm cavity and their parent vascular structures. Since these conditions represent a set of parameters that is individual for each patient, a tailored approach to optimize corrective flow measures is likely required to achieve optimal lasting corrections. Such personalized treatment planning requires postprocessing of medical imaging data to help translate the gained information in appropriate medical device use. Medical informatics provides thus the platform allowing for converging medical imaging information in better medical device use and construction.

@neurIST (www.aneurist.org), a large FP6 European IST (Information Society Technology) Integrated Project that aims at integrating biomedical informatics for the management of cerebral aneurysms, will be introduced to our community during ICS`06. This visionary project will become part of a larger international initiative, the IUPS Physiome, building on the concept of holistic modelling of organ systems - a project type that will be furthered also in future European projects (framework programme - FP7) and where neuroimaging will contribute and participate even more. @neurIST will transform the management of cerebral aneurysm by providing new insight, personalized risk assessment and methods for the design of improved medical devices and treatment protocols by integrating 3D morphological and physiological information. 
Current status and perspectives to future developments in the accordingly concerned fields of medical imaging, medical informatics and medical device industry will be on display. A focus is to invite for exploring the potential of medical informatics as enabling technology allowing for converging these to two main industrial sectors of medical imaging and medical devices. The ICS meeting has shown itself to be an increasingly interesting forum for this new approach of treating cerebrovascular intracranial diseases - a forum that benefits largely from contributions made by PhD's from different sciences. To increase the access to medical informatics, to enhance the visibility of the potential given by numerical simulations, and to initiate a validation platform, ICS'06 will launch a Virtual Intracranial Stenting Challenge (VISC), an initiative that will present its result at the next ICS'07 in Kyoto, Japan - April 18-20, 2007.

\section{CNS tissue characterization.}

The ESNR 06, 31st Congress will run from September 14 - 16, 2006. ESNR 06 will dedicate to current available techniques of CNS tissue characterization and will provide the opportunity to have complementary sessions on diagnostic imaging of pediatric, head-and-neck as well as spinal diseases.

The second meeting day will be devoted to the issue of CNS tissue characterization by imaging methods. Here sessions will develop understanding on the advanced utilization of MR techniques such as Diffusion, Perfusion, Spectroscopy as well as Molecular Imaging Techniques; indeed it is very often the integrative use of these techniques that allow us to develop a model for understanding both anatomy and function of the central nervous system in vivo.

Naturally the role of MRI Imaging and Contrast Material Companies is here on the rise and again the role and potential of converging two industrial partner technologies to enhance their joint role in imaging and understanding not only morphology but also physiology and pathophysiology will likely be discussed.

The next days, focused sessions will be devoted to Pediatric Neuroradiology, Spinal Imaging and Spinal Interventions as well as Head and Neck Imaging. These sessions will be grouped around teaching lectures held by European and international specialists and will be followed by the scientific presentations of these fields.

And more minimally invasive treatment issues: Complementary to ICS 06, image guided minimally invasive treatment (MIT) of the carotid artery (CAS - Carotid Artery Stenting) and the spine (Vertebroplasty) will be addressed and presented in form of sessions and in form of hands-on workshops.

And there are two additional teaching tools for ESNR / ICS:

We will introduce the EPOS (electronic poster) format to ESNR - a format already known to most radiologists that are attending ECR. This is the first time that the EPOS system is being tried out at the ESNR and we wish to evaluate if this format is found interesting by the congress attendees.

A further focus is on learning practical aspects of minimal invasive treatment addressed with hands-on Workshops for procedures such as involving stenting (ICS, CAS), coiling, and vertebroplasty. These workshops will involve different methods such as using in-vitro analog models (Elastrat) or full numeric simulators with haptic feedback capabilities (Cordis) or ex-vivo anatomic materials (vertebroplasty). Here we are very grateful to all that help to make this happen and that are willing to evaluate usefulness of such efforts.

Also specific lunch and coffee break symposia have been organized for and by our partners in the industry. This together with small specialized hands-on workshops should allow participants to get exposure to new technologies and to interact directly with the developers. Without the active cooperation of our industry partners the progress and the teaching in our fields as made available at this meeting would not have been possible.

The Organizing Committee wishes therefore to acknowledge the role and the support provided by national and international companies.

We would like herewith to express our best thanks to everybody who has helped to plan, organize and support the meeting. We also wish to thank to all attendees that have decided to participate actively by submitting many abstracts for oral or electronic poster presentations. 
Last but not least, we wish to congratulate and welcome the following persons for their elections to be

\title{
Honorary member of
}

\author{
ESNR: \\ G. Guglielmi, L. Picard \\ SSNR: $\quad$ M. Leonardi, C.M. Strother, J. Valk
}

\section{Honorary presidents of}

ESNR: J. Théron

ICS: $\quad$ J.-J. Merland

With best thanks and wishing a great meeting at the shores of Geneva - for the organizing committee ESNR / ICS / SSNR '06,

Yours sincerely

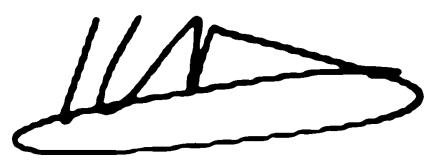

Karl-Olof Lovblad, MD

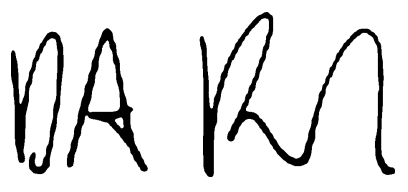

Daniel A. Rüfenacht, MD 
